\title{
Second Order Model for Strongly Sheared Compressible Turbulence
}

\author{
H. Marzougui ${ }^{\dagger}$, F. Radhia, Z. Jihene and T. Lili \\ Départemnt de Physique, Faculté des sinces de tunis campus universitaire El Manar 1060, Tunis, Tunisie
}

$\dagger$ Corresponding Author Email: hamed_merzougui@yahoo.com

(Received July 22, 2013; accepted January 16, 2014)

\begin{abstract}
In this paper, we propose a model designed to describe a strongly sheared compressible homogeneous turbulent flows. Such flows are far from equilibrium and are well represented by the $A_{3}$ and $A_{4}$ cases of the DNS of Sarkar. Speziale and $\mathrm{Xu}$ developed a relaxation model in incompressible turbulence able to take into account significant departures from equilibrium. In a previous paper, Radhia et al. proposed a relaxation model similar to that of Speziale and $\mathrm{Xu}$.This model is based on an algebraic representation of the Reynolds stress tensor, much simpler than that of Speziale and $\mathrm{Xu}$ and it gave a good result for rapid axisymetric contraction. In this work, we propose to extend the Radhia et al's. model to compressible homogenous turbulence. This model is based on the pressure-strain model of Launder et al., where we incorporate turbulent Mach number in order to take into account compressibility effects. To assess this model, two numerical simulations were performed which are similar to the cases $A_{3}$ and $A_{4}$ of the DNS of Sarkar.
\end{abstract}

Keywords: Turbulence, Homogeneous shear flow, Compressible effects, Relaxation model.

\section{INTRODUCTION}

The principal effects of compressibility on turbulence are observed in the works of Blaisdell et al. (1993) and Sarkar (1995) for homogeneous turbulent shear flows and in the works of Goebel and Dutton (1991) and Vreman et al. (1996) for turbulent mixing layer. These effects appear clearly in the reduction of the temporal growth rate of the turbulent kinetic energy and in that of the mixing layer thickness. To express these effects, the first theoretical approaches are focused on the assessment of turbulent kinetic energy. Sarkar et al. $(1991,1992)$ and Zeman $(1990,1991)$ proposed algebraic models to explain the explicit dilatational terms such as the pressure-dilatation $\pi_{d}=\overline{p^{\prime} d^{\prime}}$ and the dilatational dissipation $\varepsilon_{d}$. These models perform well in predicting, at low turbulent Mach number, the compressibility effects on turbulence. However when compressibility is higher, Sarkar (1995) and Vreman et al. (1996) confirmed that these terms can not be regarded as essential in causing the reduced growth rate. They also pointed out that compressibility was found to affect the production term via the pressure-strain correlations. Consequently, the compressibility effects must be required in these terms. Today, no model for the pressure-strain correlation based on the integration of the complete Poisson's equation has been published. Nevertheless, proposed extensions of incompressible models were made in particular configurations. We quote here the works of Cambon et al. (1993), Vreman et al. (1996), Marzougui et al. (2005) and Huang et al. (2008). The application of Marzougui et al.'s model shows a good agreement with DNS results of Sarkar (1995) for cases $A_{1}$ and $A_{2}$ which correspond to a moderate mean shear rate so that nonlinear effects are important. However, notable deviations have been observed in cases $A_{3}$ and $A_{4}$. Using the turbulent length scale defined by Blaisdell, Simone et al. (1997) showed that the initial shear number $S k / \varepsilon_{S}$ for $\operatorname{cases} A_{3}$ and $A_{4}$ takes respectively the following values: 8.25 and 16.5 . They noted that these cases could be treated within the framework of rapid distortion theory. It is shown that second-order closure models- as they have commonly been formulated since Launder et al.- perform poorly in the prediction of such flows, because these models are based on strong assumptions such as equilibrium isotropic turbulence. To describe better the turbulent flows presenting a localized regions of large shear rates , it is important to develop models having satisfactory performances for significant departures from equilibrium. The incompressible model suggested by Speziale and Xu (1996) seems to meet these needs. Despite the improvements made by this model, this one is few used by the researchers. This is due to the complexity of the algebraic representation of the Reynolds stress tensor adopted by these authors in their model. In order to stress the effectiveness of this type of 
model, Radhia et al. (2010) proposed a relaxation model similar to that of Speziale and Xu. This model is based on an algebraic representation of the Reynolds stress tensor which is much simpler than that proposed by Speziale and $\mathrm{Xu}$. In the present work, we propose to extend the Radhia et al.'s model on compressible homogeneous turbulent flows. We start by presenting, briefly, the relaxation model for compressible homogeneous turbulent flows. In this model, the dilatational terms are given by the Sarkar's models. For the pressure-stain correlation, we propose a compressible correction parameterized according to the turbulent Mach number. To evaluate the proposed model, we take the two cases $A_{3}$ and $A_{4}$ of the DNS of Sarkar (1995).

\section{Presentation of the Relaxation MODEL}

\subsection{Governing equations}

The homogeneity hypothesis with a constant mean velocity gradient for compressible fluid appears as an additional condition that the average density remains constant in space and time and the statistical average of Reynolds and Favre are identical. This allows the extension of the incompressible models on compressible turbulent flows. Thus, the model that we are proposing results from a simple transposition of the incompressible model of Radhia et al. This model expresses the $b_{i j}$ components of the anisotropy tensor as follows:

$$
\frac{d}{d t} b_{i j}=-C_{r} \frac{\varepsilon_{s}}{k}\left(b_{i j}-b_{i j}^{e}\right)
$$

$C_{r}$ is the relaxation coefficient, it is given by:

$$
C_{r}=1+0.9 \frac{k}{\varepsilon_{s}}\left(\tilde{S}_{i j} \tilde{S}_{i j}\right)^{1 / 2}
$$

$\mathrm{k}$ and $\varepsilon_{s}$ represent the turbulent kinetic energy and its solenoidal dissipation rate, respectively. These quantities are given by the following equations :

$$
\begin{gathered}
\frac{d}{d t} k=-2 k\left(b_{i j}+\delta_{i j} / 3\right) \tilde{u}_{i, j}-\varepsilon_{s} \\
-\left(\frac{p^{\prime} d^{\prime}}{\bar{\rho}}+\varepsilon_{d}\right) \\
\frac{d}{d t} \varepsilon_{s}=-2 C \varepsilon_{1} \varepsilon_{s}\left(b_{i j}+\delta_{i j} / 3\right) \tilde{u}_{i, j} \\
-C \varepsilon_{2} \frac{\varepsilon_{s}^{2}}{k}
\end{gathered}
$$

$\bar{p} d^{\prime}$ and $\varepsilon_{d}$ represent the dilatational terms. In the present work, these terms are given by the Sarkar's models $(1991,1992)$ :

$\varepsilon_{d}=\alpha M_{t}^{2} \varepsilon_{s}$ $\overline{p^{\prime} d^{\prime}}=-\alpha_{2} \bar{\rho} P M_{t}+\alpha_{3} \bar{\rho} \varepsilon_{s} M_{t}^{2}$

$C_{\varepsilon_{1}}$ and $C_{\varepsilon_{2}}$ are constants that are taken to be 1.44 and 1.95 , respectively.

$b_{i j}^{e}$ are the equilibrium values of the components of the anisotropy tensor which are given by the following algebraic representation:

$$
\begin{aligned}
b_{i j}^{e} & =\beta_{1} \tilde{S}_{i j}+\beta_{2}\left(\tilde{S}_{i k} \tilde{\Omega}_{k j}-\tilde{\Omega}_{i k} \tilde{S}_{k j}\right) \\
& +\beta_{3}\left(\tilde{S}_{i k} \tilde{S}_{k j}-\frac{1}{3} \tilde{S}_{m n} \tilde{S}_{m n} \delta_{i j}\right)
\end{aligned}
$$

$\tilde{S}_{i j}$ and $\tilde{\Omega}_{i j}$ denote the mean strain and the mean vorticity, respectively

$$
\tilde{S}_{i j}=\frac{1}{2}\left(\tilde{u}_{i, j}+\tilde{u}_{j, i}\right), \tilde{\Omega}_{i j}=\frac{1}{2}\left(\tilde{u}_{i, j}-\tilde{u}_{j, i}\right)
$$

the terms $\beta_{i} \quad(\mathrm{i}=1,2,3)$ are scalar functions of the invariants $\eta_{1}=\tilde{S}_{i j} \tilde{S}_{i j}$ and $\eta_{2}=\tilde{\Omega}_{i j} \tilde{\Omega}_{i j}$.

$\beta_{1}=\frac{\left(\frac{d k}{k d t}+C_{1} \frac{\varepsilon}{k}\right)\left(C_{2}-4 / 3\right)}{D\left(\eta_{1}, \eta_{2}\right)}$

$$
\begin{aligned}
& \beta_{2}=-\frac{\left(C_{4}-2\right)\left(C_{2}-4 / 3\right)}{D\left(\eta_{1}, \eta_{2}\right)} \\
& \beta_{3}=\frac{2\left(C_{3}-2\right)\left(C_{2}-4 / 3\right)}{D\left(\eta_{1}, \eta_{2}\right)} \\
& D\left(\eta_{1}, \eta_{2}\right)=\left(\frac{d k}{k d t}+C_{1} \frac{\varepsilon_{s}}{k}\right)^{2}-\frac{2}{3}\left(C_{3}-2\right)^{2} \eta_{1} \\
& \quad+2\left(C_{4}-2\right)^{2} \eta_{2}
\end{aligned}
$$

the $C_{i} \quad(\mathrm{i}=1,2,3,4)$ are the constants of the modified model for the pressure strain correlation. The general form of this model is :

$$
\begin{aligned}
& \Pi_{i j}=-\bar{\rho} C_{1} \varepsilon b_{i j}+C_{2} \bar{\rho} k \tilde{S}_{i j} \\
& +C_{3} \bar{\rho} k\left[b_{i k} \tilde{S}_{j k}+b_{j k} \tilde{S}_{i k}-\frac{2}{3} b_{m n} \tilde{S}_{m n} \delta_{i j}\right] \\
& +C_{4} \bar{\rho} k\left[b_{i k} \tilde{\Omega}_{j k}+b_{j k} \tilde{\Omega}_{i k}\right]
\end{aligned}
$$

\subsection{Modeling of compressibility effects on pressure-} strain correlation.

The compressibility effects on homogeneous turbulence are parameterized by two parameters: the turbulent Mach number $M_{t}$ and the gradient Mach number $\mathrm{M}_{\mathrm{g}}$. The turbulent Mach number is the ratio of the acoustic time 
scale to the turn-over time : $M_{t}=\tau_{a} / \tau_{t}$. The gradient Mach number is defined by Sarkar (1995), Durbin et al. (1992) and Cambon et al. (1993) as being the ratio of the acoustic time scale to the mean distortion time scale : $M_{g}=\tau_{a} / \tau_{d}=\alpha \frac{s k}{\varepsilon} M_{t}$. The direct numerical simulations of Sarkar (1995) for homogeneous turbulent shear flows and those of Vreman et al. (1996) for turbulent mixing layer show that the structural effects of compressibility must be modeled in the pressure-strain correlation. This result was confirmed in several studies thereafter. We have just mentioned in the introduction that there is no trace of modeling of the pressure-strain correlation in compressible turbulence based on the complete Poisson's equation and the existing of compressible models are extensions of models established already in incompressible mode. We quote, for example, the works of Cambon et al. (1993), Vreman et al. (1996), Marzougui et al. (2005) and Huang et al. (2008)

\section{- Cambon et al.'s model. (1993)}

The obtained results from the RDT of Cambon et al. for an axial compression show that the correlations between the turbulent pressure field and the turbulent velocity field decrease in a monotonous way according to the distortion Mach number. This observation led Cambon et al. to develop a compressible correction to the standard model of Launder et al. (1975).

$$
\Pi_{i j}=\left(\Pi_{i j}^{r}\right)_{i n c} \exp -\left(M_{d} / C_{d m}\right),
$$

the slow terms are neglected by Cambon et al. because it is about a rapid deformation.

\section{- Vreman et al.'s model. (1996)}

The direct numerical simulations of Vreman et al. for turbulent mixing layer show that the contribution of the pressure-stain correlation decreases with the increase of compressibility. These simulations enable them to propose a compressible correction of the axial component of the rapid part of pressure-strain correlation. Their model is written:

$$
\Pi_{11}=\left(\Pi_{11}^{r}\right)_{i n c} f\left(M_{r}\right)+\left(\Pi_{11}^{S}\right)_{i n c},
$$

where $f\left(M_{r}\right)$ is a related function. It is a function of the relative Mach number.

\section{- Marzougui et al.'s model (2005)}

The contribution of Marzougui et al. appears in the correction of the $C_{i}$ coefficients, which became in compressible turbulence functions of the turbulent Mach number. The suggested method is based on proportionality relations between the ratio of compressible and incompressible components of the pressure-strain correlation and the ratio relating the compressible and incompressible growth rate of the turbulent kinetic energy. This method generates a pressure strain model parameterized according to the turbulent Mach number.

$$
\begin{aligned}
& \Pi_{i j}=-C_{1}^{I} \frac{\left(1-0.44 M_{t}^{2}\right)^{2}}{\left(1+\alpha M_{t}^{2}\right)} \bar{\rho} \varepsilon_{s} b_{i j}+C_{2}^{I} \bar{\rho}_{k} \tilde{S}_{i j} \\
& +C_{3}^{I}\left(1-1.5 M_{t}^{2}\right) \bar{\rho} k\left(b_{i k} \tilde{S}_{j k}+b_{j k} \tilde{S}_{i k}\right. \\
& \left.-\frac{2}{3} b_{m n} \tilde{S}_{m n} \delta_{i j}\right) \\
& +C_{4}^{I}\left(1-0.5 M_{t}\right) \bar{\rho} k\left(b_{i k} \tilde{\Omega}_{j k}+b_{j k} \tilde{\Omega}_{i k}\right)
\end{aligned}
$$

\section{- Huang and Fu's model (2008)}

The compressible model proposed by Huang and $\mathrm{Fu}$ for the pressure-strain correlations is as follow:

$$
\begin{aligned}
& \Pi_{i j}=-C_{1} \varepsilon b_{i j}+C_{2} k\left(\tilde{S_{i j}}-\frac{1}{3} \tilde{S}_{k k}\right) \\
& +C_{3} k\left(b_{i l} \tilde{S}_{l j}+b_{j l} \tilde{S}_{l i}-\frac{2}{3} b_{l k} \tilde{S}_{k l} \delta_{i j}\right) \\
& +C_{4} k\left(\tilde{\Omega}_{i l} b_{l j}-b_{i l} \tilde{\Omega}_{l j}\right)
\end{aligned}
$$

The coefficients of this model are:

$$
\begin{aligned}
& C_{1}=3.6, C_{2}=0.8, C_{3}=1.2+2 f\left(M_{t}\right), \\
& C_{4}=1.2-2 f\left(M_{t}\right)
\end{aligned}
$$

Where $f\left(M_{t}\right)$ is a function of the turbulent Mach number: $f\left(M_{t}\right)=0.25 \exp \left(-0.05 / M_{t}^{3}\right)$

It is clear when $f\left(M_{t}\right)=0$, the model is the same as the Gibson and Launder's model (1978). $\varepsilon$ is the total turbulent dissipation. The Huang and Fu's model (2008) for the dilatational part of turbulent dissipation is algebraic and takes the following form:

$\varepsilon_{d}=0.1\left(1-\exp \left(-5 M_{t}^{2}\right)\right)$

These works gave us the idea to introduce a damping function into the incompressible model of Launder et al. The compressibility parameter used is the turbulent Mach number. Our proposal is based on the work of Pantano and Sarkar (2002). These authors, by direct numerical simulations for shear layers, proposed a damping function into the deviatoric part of the pressure-strain correlation of the form:

$$
\begin{aligned}
& f\left(M_{c}, M_{g}, M_{t}\right)=1-b_{1} M_{g}^{2}+b_{2} M_{t}^{2}+b_{3} M_{g} M_{t} \\
& +b_{3} M_{g} M_{t}, \text { low } M_{c} \\
& f\left(M_{c}, M_{g}, M_{t}\right)=c+(1-c)
\end{aligned}
$$




$$
\frac{1+a M_{c}^{2} \exp -\left(2 M_{c}-0.5\right)^{2}}{1+b M_{c}^{2}}, \text { large } M_{c}
$$

In the present study, we are interested in the turbulent flows where the compressibility is significant. Consequently our choice falls on the exponential form of $f\left(M_{c}\right)$. Let us recall that the analogies established by Sarkar between homogeneous turbulent shear flow and the range around the mid-plane of mixing layer lead to the relation: $M_{c} \sim 0.46 M_{g}$, where $M_{g} \sim \frac{S k}{\varepsilon} M_{t}$. The parameters $M_{g}$ and $M_{t}$ are proportional when we admit the assumption that, for large times, the shear number $S k / \varepsilon_{S}$ reaches an asymptotic value. This assumption leads us to rewrite the damping function using the turbulent Mach number.

$$
H\left(M_{t}\right)=\frac{1+a_{1} M_{t}^{2} \exp -\left(3 M_{t}-0.5\right)^{2}}{1+a_{2} M_{t}^{2}}
$$

$a_{1}$ and $a_{2}$ are constants which take respectively the following values: 8.4, 3.8. According to the writing $\Pi_{i j}=H\left(M_{t}\right)\left(\Pi_{i j}\right)_{i n c}$, the $C_{i}$ Constants in the relations $(8,9,10)$ take the form: $C_{i}=C_{i}^{i n c} H\left(M_{t}\right)$. $C_{i}^{i n c}$ are the constants of the incompressible model of Launder et al. (1975).

\section{Application Of THE Present Model FOR COMPRESSIBLE HOMOGENEOUS TURBULENT SHEAR FLOW}

The transport equations (1), (3) and (4) incorporating our model were solved numerically for compressible homogeneous turbulent shear flow using a fourthorder accurate Runge-Kutta numerical integration scheme. Comparisons will be made with Hung and Fu's model and with the DNS of Sarkar. The direct numerical simulations of Sarkar show that the influence of the gradient Mach number on the reduction of the temporal growth rate of the turbulent kinetic energy is more significant than that of the turbulent Mach number. These results led Sarkar as well as other researchers to consider that $\mathrm{Mg}$ is the good parameter in order to study the compressibility effects on turbulence. In the direct numerical simulations of Sarkar (1995), the definition of the gradient Mach number is built on the integral length scale in the transverse direction. This choice leads to low values of the gradient Mach number (table 1).

Table 1 Initial conditions

\begin{tabular}{ccc}
\hline Case & $\left(M_{g, 0}\right)$ Sarkar & $\left(M_{g, 0}\right)$ Simone et al \\
\hline $\mathrm{A}_{3}$ & 0.66 & 6.6 \\
$\mathrm{~A}_{4}$ & 1.32 & 13.2 \\
\hline
\end{tabular}

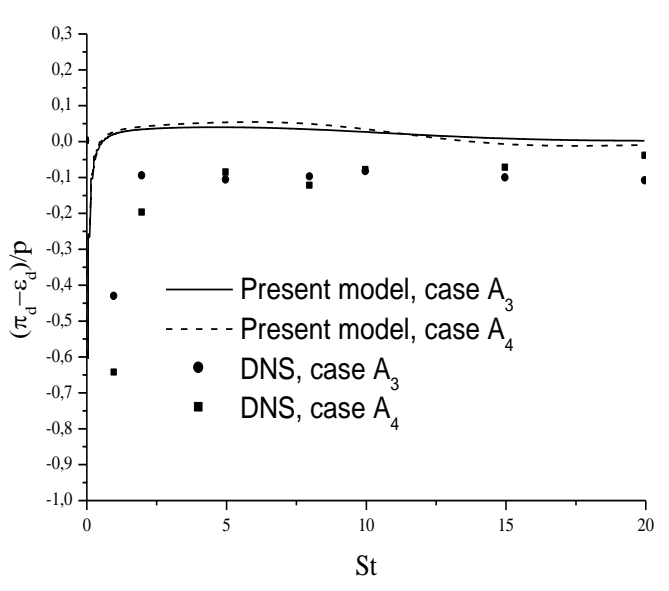

Fig. 1. the evolution of the dilatational terms

The two simulations $A_{3}$ and $A_{4}$ are taken again by Simone et al. (1997). They defined the gradient Mach number using the length scale $l=(2 k)^{3 / 2} / \varepsilon_{s}$, which conducts to large values of $M_{g}$ and $S k / \varepsilon_{S}$ (table1). From these values they emphasized that $A_{3}$ and $A_{4}$ must be studied with the rapid distortion theory.

First, we shall consider model predictions when the damping function $H\left(M_{t}\right)=0$ in order to assess the performance of the proposed correction to the L.R.R's model for the pressure-strain correlation. Figure 2 to Fig 7 show that the Radhia et al's model with the dilatational dissipation and the pressure-dilatation models of sarkar is unable to predict the dramatic change in the anisotropy of turbulence that arises from compressibility. This means that for high turbulent Mach number, the dilatational terms are smaller to explain the compressibility effects on the turbulence. This is can be seen clearly in Fig 1, which represents the time evolution of the dilatational terms normalized by the production term $P=-2 k b_{12} S$.

This implies that the dilatational terms cannot be regarded as essential in causing the reduced growth rate of the turbulent kinetic energy observed in DNS results. The time variation of the Reynolds stress anisotropies $b_{11}, b_{22}$ and $b_{12}$ are plotted in Fig 1, Fig 2, Fig 3 and Fig 4 respectively. It is clear, from these results, that the agreement between our model predictions and the DNS of Sarkar is good. The Hung and Fu's model does a reasonably good job in predicting the time evolution of $b_{12}$.However, it yields considerably different results for the normal components $b_{11}$ and $b_{22}$. About the compressibility effects on the anisotropy of the Reynolds stress tensor, we observe that an increase in the gradient Mach number involves an increase in the normal components $b_{11}, b_{22}$ and a reduction in the tangential 

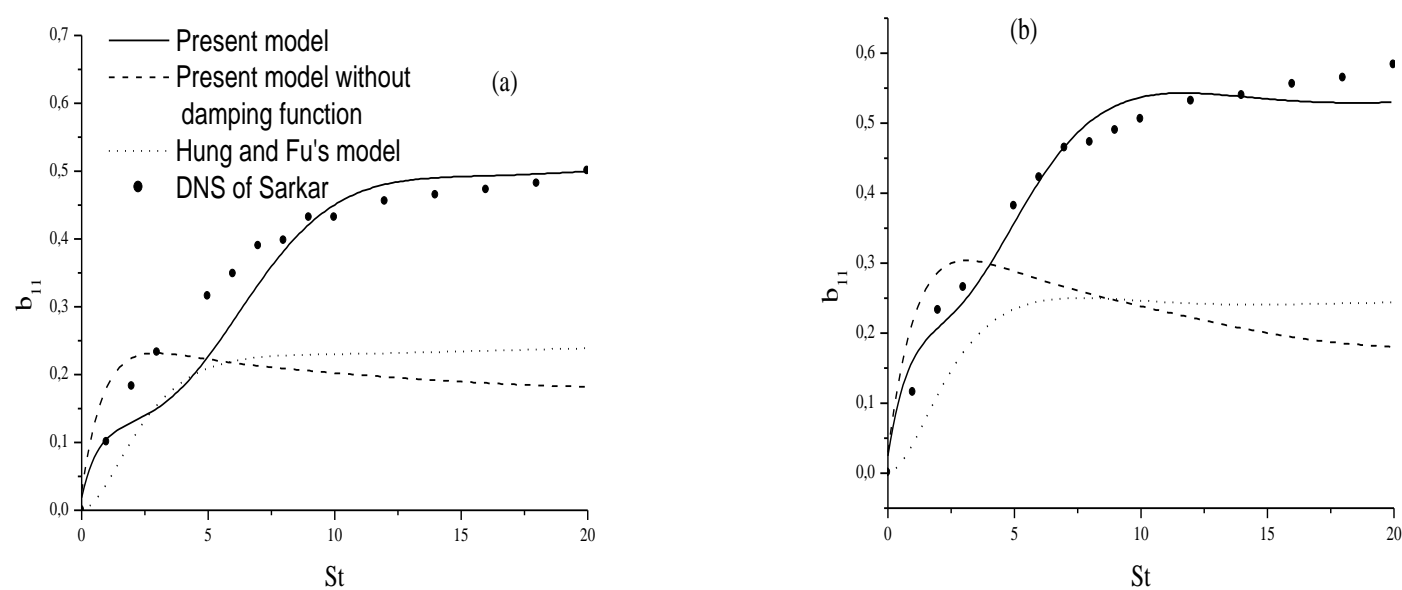

Fig. 2. The evolution of streamwise Reynolds stress anisotropy $b_{11}:$ (a) case $A_{3}$, (b) case $A_{4}$
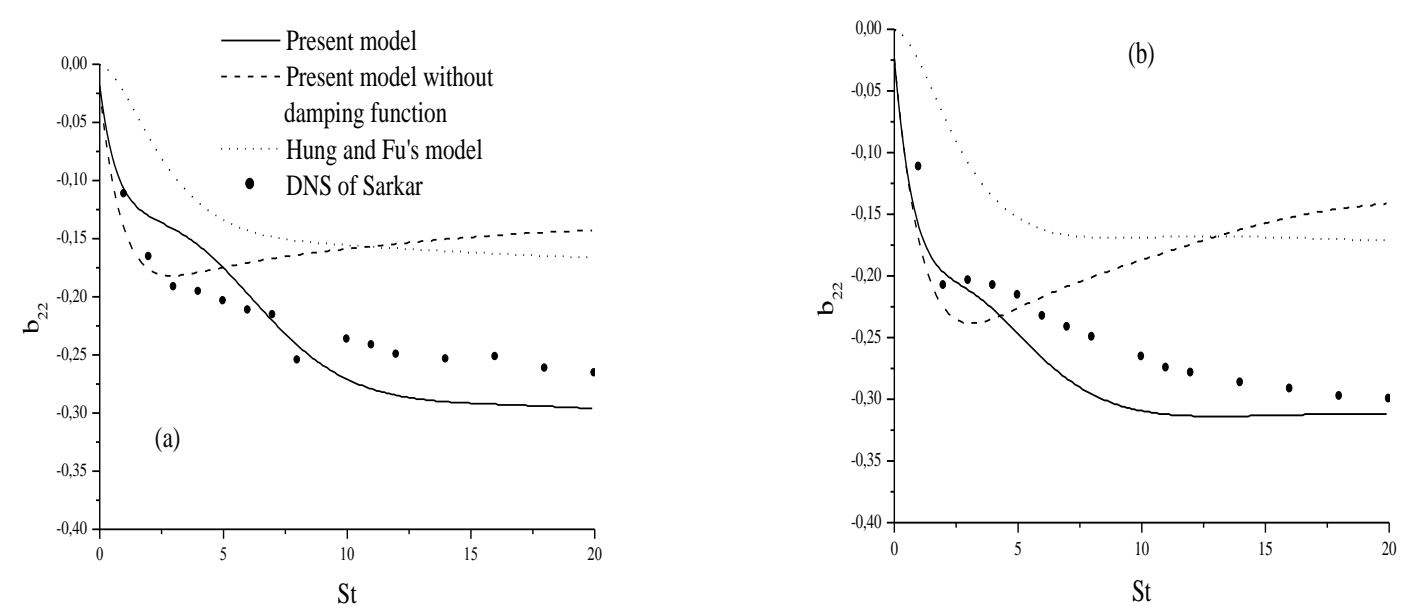

Fig. 3. The evolution of transverse Reynolds stress anisotropy $b_{22}$ :(a) case $A_{3}$, (b) case $A_{4}$
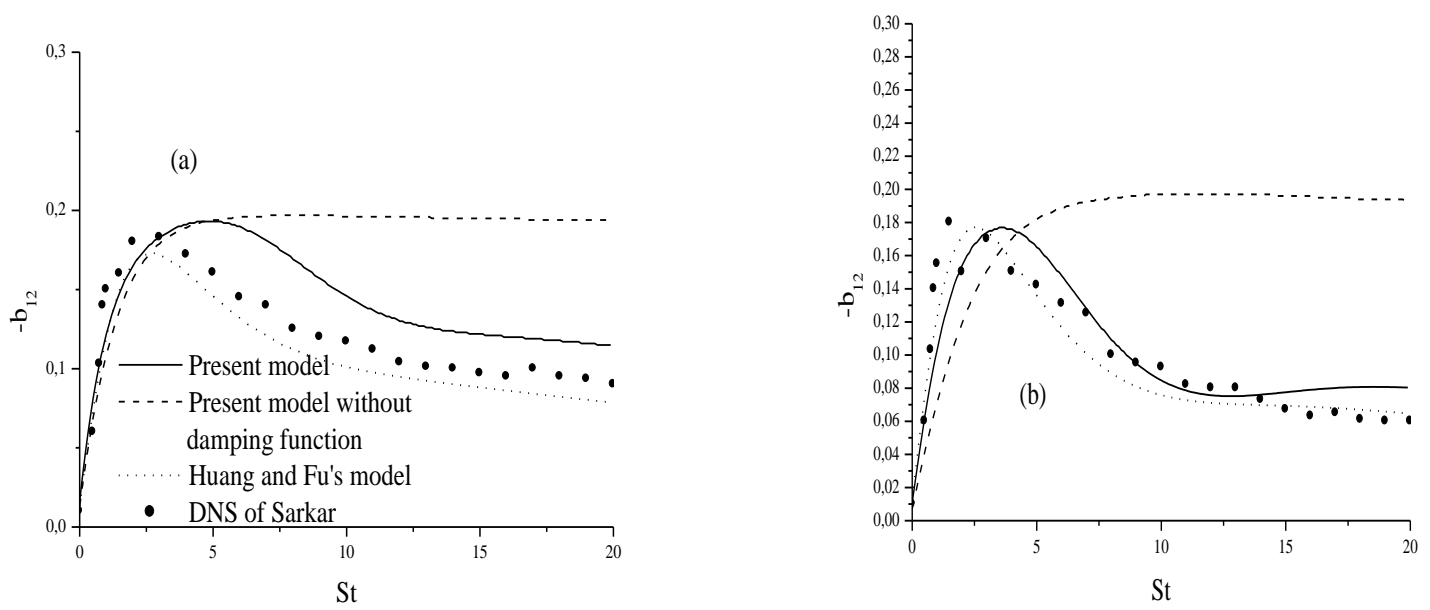

Fig. 4. The evolution of Reynolds shear stress anisotropy $b_{12}$ : (a) case $A_{3}$, (b) case $A_{4}$

component $b_{12}$ at the end of simulations. These figures show also that after a transitional phase, the components $b_{i j}$ of the anisotropy tensor converge towards asymptotic states which depend on the initial conditions. This point is the subject of the last part of this section.

Figures 5, 6 and 7 show the time evolution of the components $\Pi_{11}, \Pi_{22}$ and $\Pi_{12}$ of the pressure-stain tensor. In comparison with the DNS results of Sarkar, the proposed model reproduces in a correct way the action which compressibility exerts on the pressure-strain correlations. This action appears by the inhibition of the pressure fluctuating field when the gradient Mach number increases. The Hung and Fu's model predicts the $\Pi_{11}$ and $\Pi_{22}$ components reasonably. For the $\Pi_{12}$, 

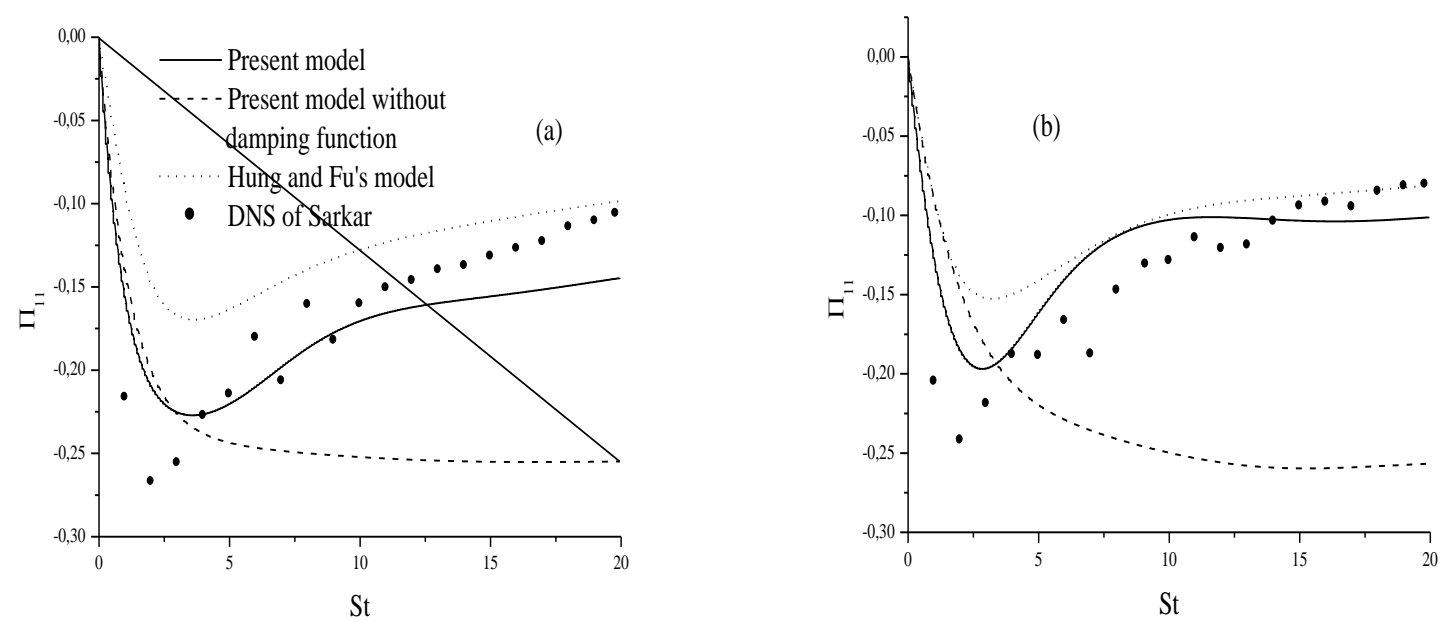

Fig. 5. The evolution of pressure-strain $\Pi_{11}$ : (a) case $A_{3}$, (b) case $A_{4}$
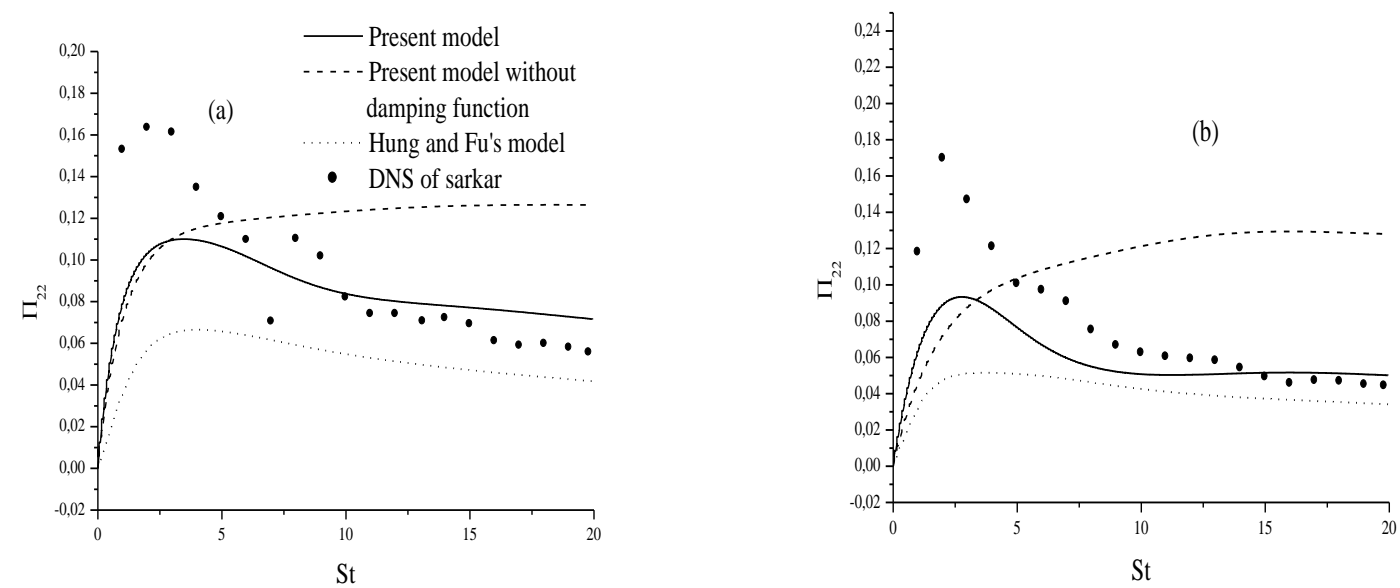

Fig. 6. The evolution of pressure-strain $\Pi_{22}$ : (a) case $A_{3}$, (b) case $A_{4}$
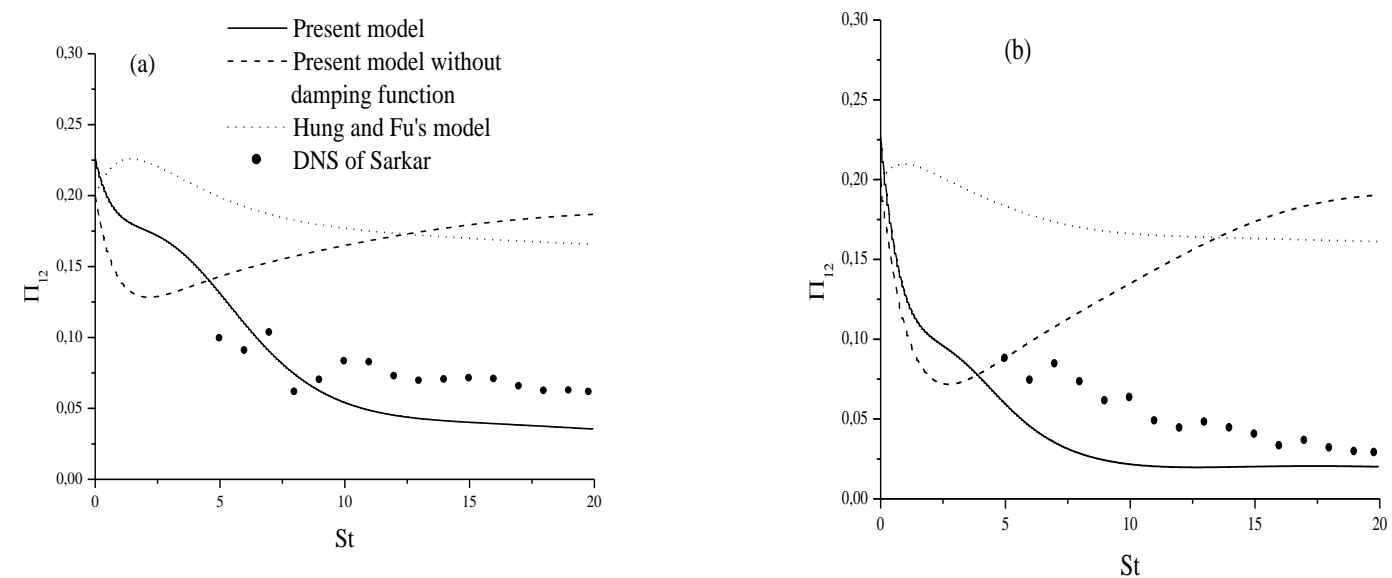

Fig. 7. The evolution of pressure-strain $\Pi_{12}$ :(a) case $A_{3}$, (b) case $A_{4}$

this model over-predicts the DNS. We know that the principal role of these correlations is the redistribution of the energy produced in the principal direction of the flow on the components of the Reynolds tensor. These terms are strongly reduced with the increase in $\mathrm{M}_{\mathrm{g}}$. Consequently, a significant quantity of the kinetic energy remains stored in the flow direction. This makes it possible to explain the strong amplification of $b_{11}$ observed in the DNS of Sarkar when Mg increases. The time evolution of the turbulent Mach number $M_{t}$ predicted by our model is shown in Fig 8 along with the predictions of the Hung and Fu's model and the DNS of Sarkar. It is clearly seen that both models appear to be able to predict the increase of the turbulent Mach number 

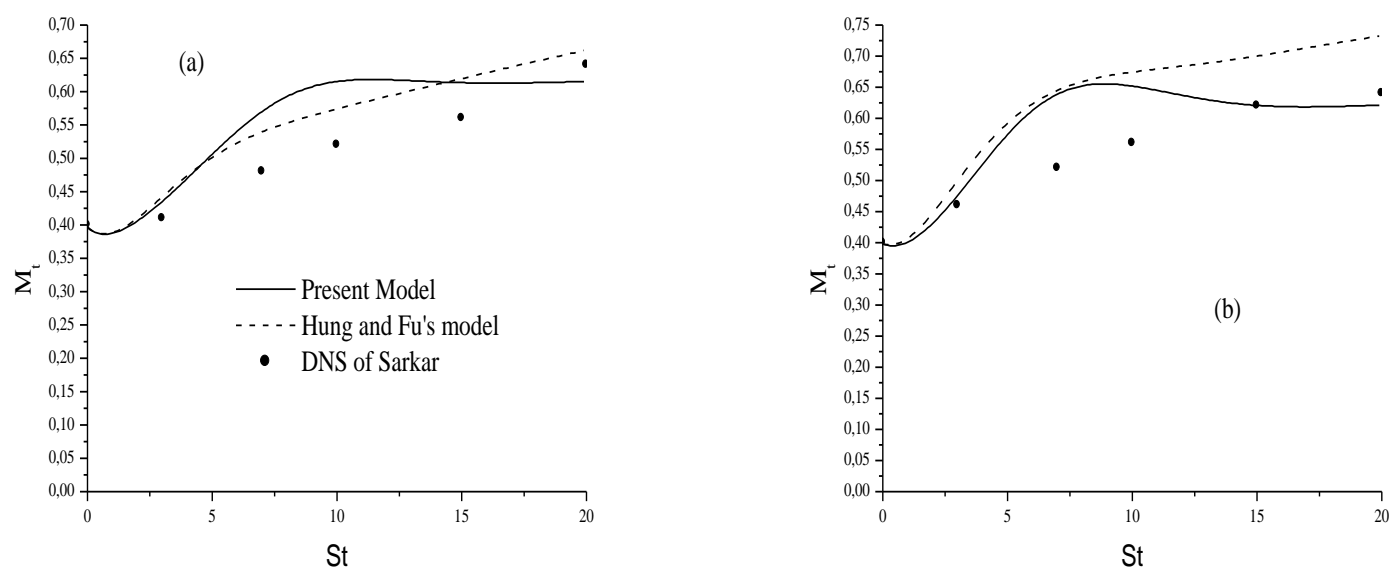

Fig. 8. The evolution of turbulent Mach number: (a) case $A_{3}$, (b) case
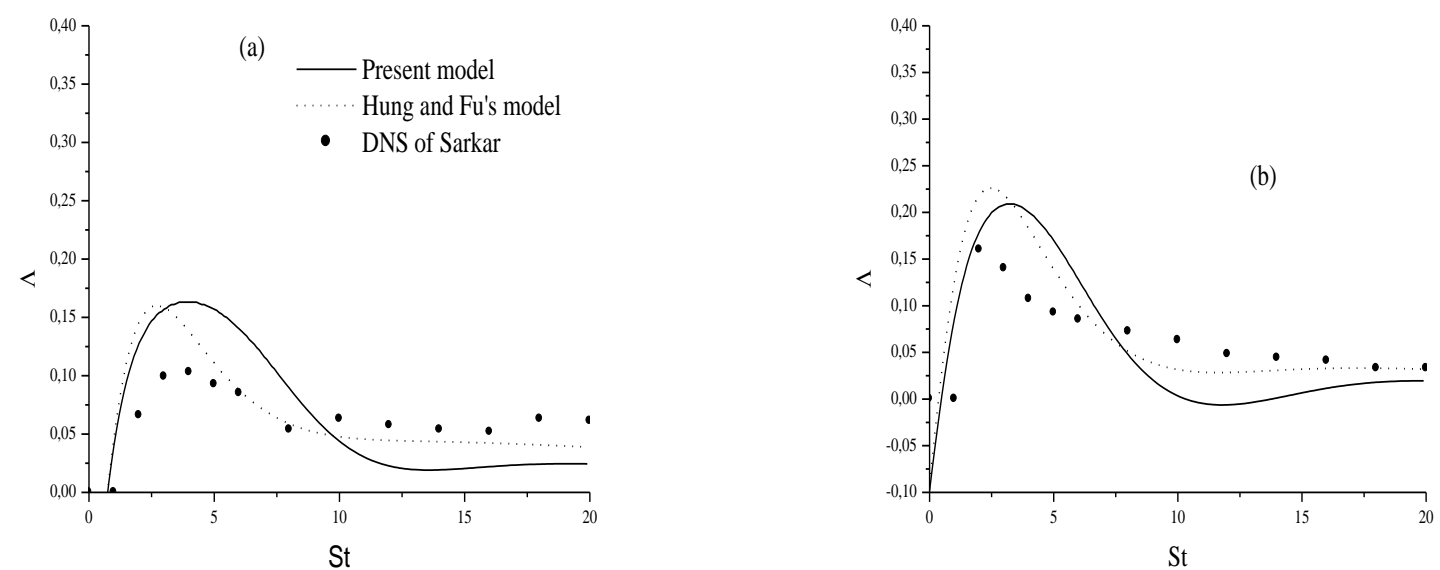

Fig. 9. The evolution of the turbulent energy growth rate: (a) case $A_{3}$, (b) case $A_{4}$

with increasing gradient Mach number. It is also observed that our model gives slightly better result than the Hung and Fu's model. In Fig 9, we plot the time evolution of the temporal growth rate of the turbulent kinetic energy $\Lambda=\frac{1}{S k} \frac{d k}{d t}$ for cases $A_{3}$ and $A_{4}$ In this evolution, two phases are distinguished. The first $(S t \leq 5)$ is characterized by a destabilizing effect of a rapid distortion-for large initial values of shear number the turbulence develops to become strongly anisotropic and the turbulent kinetic energy grows rapidly. This behavior is seen in rapidly distorted incompressible homogeneous shear flow. This trend is reversed at $S t=5$. Indeed, we observe for large times the stabilizing effect of compressibility that appears clearly in the reduction of $\Lambda$ with the increase in the gradient Mach number. This phenomenon which has often been observed in DNS results for compressible turbulent shear flows is predicted by both models (our model and the Hung et Fu's model)

Now, we will discuss the equilibrium states in compressible homogeneous shear flow. Several works concerning the incompressible homogeneous turbulent shear flows have shown that the quantities $b_{i j}, \Pi_{i j}$ and $\Lambda$ evolve, for large times, to "universal" asymptotic values which do not depend on the initial conditions. The question of the existence of asymptotic values arises with the works of Blaisdell et al. on compressible homogeneous turbulent flows.

The numerical simulations carried out by these authors showed that, when the compressibility is low, the turbulent Mach number continues to grow with time. This work led several authors to consider that compressible turbulence cannot evolve to an asymptotic state. The direct numerical simulations of Sarkar (1995) show that if compressibility is significant, the evolution curves of the statistical quantities $b_{i j}, \Pi_{i j}$ and $\Lambda$ have a trend to be flattened for large times. This work leads several researchers to start again the debate about the existence of asymptotic states for homogeneous compressible turbulent flows. Zeman (1992) already postulated an asymptotic value for the turbulent Mach number. He supposes that an equilibrium between fluctuations speed and speed of sound is established due to the formation of eddy shocklets. The turbulent Mach number equation developed by Zeman (1997) shows that the evolution of this number is controlled by $b_{12}$ and $Y_{p}$ (energetic terms). This equation is written :

$$
\begin{aligned}
& \frac{d}{d t} M_{t}=M_{t} b_{12}\left[\left(Y_{p}-1\right)\right. \\
& \left.+\gamma(\gamma-1) M_{t}^{2} / 2\right]
\end{aligned}
$$


Table 2 Comparison of the present model predictions for the asymptotic values of the anisotropy tensor for cases $A_{3}$ and $A_{4}$

\begin{tabular}{|c|c|c|c|c|}
\hline Asymptotic values & Present Model & Hung and Fu's model & DNS & Formulas of Stefan \\
\hline$b_{11, \infty}$ & $0.499 ; \quad 0.54$ & $0.239 ; \quad 0.244$ & $0.5 ; \quad 0.58$ & $0.504 ; \quad 0.589$ \\
\hline$b_{22, \infty}$ & $-0.296 ; \quad-0.31$ & $-0.166 ;-0.171$ & $-0.266 ;-0.3$ & $-0.264 ; \quad-0.3$ \\
\hline$b_{12, \infty}$ & -0.11 & $-0.078 ; \quad-0.0648$ & $-0.09 ;-0.06$ & $-0.093 ;-0.056$ \\
\hline
\end{tabular}

The asymptotic solutions of this equation are:

$$
\begin{aligned}
& M_{t, \infty}=0, \\
& M_{t \infty}=\left[\left(\frac{2}{\gamma(\gamma-1}\right)\left(\frac{1-C_{\varepsilon_{2}}}{1-C_{\varepsilon_{1}}}-1\right)\right]^{1 / 2}
\end{aligned}
$$

The first solution corresponds to the incompressible limit. The solution in compressible mode is constant. These observations indicate that the asymptotic value of $M_{t}$ does not depend on the initial conditions. This point is in agreement with the assumptions of Zeman (1992) and Sarkar (1995) and with the results obtained by our model. Fig 8 shows that after transitional phase, $M_{t}$ converges independently of the initial conditions towards an asymptotic value of about 0.62. However, the quantities such as $b_{i j}, \Pi_{i j}$ and $\Lambda$ calculated by the proposed model converge towards asymptotic values. These values depend on the initial conditions. These trends are in agreement with the numerical simulations of Sarkar. This behavior is already confirmed by Stefan (2003). This author shows that the asymptotic values of the components $b_{11}, b_{22}$ and $b_{12}$ of the anisotropy tensor are clarified according to the gradient Mach number.

$$
\begin{aligned}
& b_{11}=\frac{2}{3}-0.4 \exp \left(-0.3 M_{g}\right) \\
& b_{22}=-\frac{1}{3}+0.17 \exp \left(-0.3 M_{g}\right)
\end{aligned}
$$

\section{REFERENCES}

G.A. Blaisdell, N.N. Mansour, and W.C. Reynolds (1993). "Compressibility effects on the growth and structure of homogeneous turbulent shear flow ", Journal of fluid Mechanics, vol. 256, p.443.

S.Sarkar, (1995). "The stabilizing effect of compressibility in turbulent shear flow", Journal of fluid Mechanics, vol. 282, p.163.

S.G.Goebel, J.C.Dutton (1991). "Experimental study of compressible turbulent mixing layers", American Institute of Aeronautics and Astronautics, vol.29, p.538.

A.W.Vreman, N.D.Sandham, K.H.Luo (1996). "Compressible mixing layer growth rate and turbulence characteristics", Journal of fluid Mechanics, vol.320, p.235. $b_{12}=-0.17 \exp \left(-0.2 M_{g}\right)$

The long-time values of $b_{i j}$ obtained by the present model, the DNS of Sarkar, the Hung and Fu's model and the formulas of Stefan are summarized in Table 2. As one can remark that the proposed model gives better results for the prediction of the equilibrium states in homogeneous turbulent shear flows.

\section{Conclusion}

In this paper, we proposed a second-order model for strongly sheared compressible turbulence. In order to include the compressibility effects, a same damping function is introduced into the slow part and the rapid part of the Launder et al.'s model for the pressure-strain correlation. It should be noted that this is one of the most important assumptions used to derive this model. The proposed model is applied to simulate compressible homogeneous turbulent shear flow. Our results are compared with the DNS of Sarkar and with those obtained by the Hung and Fu's model. From the above comparisons, we note that on the overall our model works better than the Hung and Fu's model. More detailed tests of the present model are currently underway.

S. Sarkar (1992). "The pressure dilatation correlation in compressible flows", Journal of Physics of Fluids, Vol.4, p.2674.

S. Sarkar, G. Erlebacher, M. Hussaini and H.O. Kreis (1991). "The analysis and Modeling of dilatational terms in compressible turbulence ", Journal of fluid Mechanics, Vol.227,p.473.

O.Zeman (1990). "Dilatation dissipation: the concept and application in modeling compressible mixing layers", Journal of Physics of Fluids, vol.2, p178.

O.Zeman (1991). "On the decay of compressible isotropic turbulence", Journal of Physics of Fluids,Vol.3, pp.951.

C. Cambon, G.N. Coleman, and N.N. Mansour (1993). "Rapid distortion analysis and direct simulation of 
H. Marzougui et al. / JAFM, Vol. 8, No. 1, pp. 113-121, 2015.

compressible homogeneous turbulence at finite Mach numbers", Journal of fluid Mechanics, Vol.257, p641.

H. Marzougui, H.Khlifi and T.Lili (2005). "Extension of the Launder, Reece and Rodi model on compressible turbulent shear flow", European Physical Journal B , Vol. 45,p.147.

S.Hung and S.Fu (2008). "modeling of pressure-strain correlation in compressible turbulent flow Acta Mech Sin, Vol 24, p37.

A. Simone, G.N. Coleman and C. Cambon (1997). "The effect of compressibility on turbulent shear flow: a rapid-distortion-theory and direct-numericalsimulation study", Journal of Fluid Mechanics, Vol. 330, p. 307.

C.G. Speziale, X.H. Xu (1996). "Towards the development of second-order closure models for nonequilibrium turbulents flows", International Journal of heat and Fluid Flow, vol.17, p.238.

F.Radhia, H.Marzougui, Z.Jihene, T.Lili (2010). "On a relaxation model for non-equilibrium turbulent flows", Comptes rendus .Mecanique, vol. 338, p.355.

P.A. Durbin and O. Zeman (1992). "Rapid Distortion theory for homogeneous compressed turbulence with application to modeling", Journal of fluid Mechanics, vol.242, p.349.

B.E.Launder, G.J.Reece, W.Rodi (1975). "Progress in the development of a Reynolds stress turbulence closure", Journal of fluid Mechanics,vol.68, p.537.

M. Gibson, B.E. Launder (1978). "Ground effects on pressure fluctuations in the atmospheric boundary layer", J.Fluid Mech, vol 86, p491.

C.Pantano, S. Sarkar (2002). "A study of compressibility effects in the high-speed turbulent shear layer using direct simulation", Journal of fluid Mechanics, Vol. 451, p.329.

O. Zeman (1992). "Towards a constitutive relation in compressible flows. In T.B. Gatski, S. Sarkar, and C.G. Speziale, editors, Studies in Turbulence. Springer Verlag, 1992.

Markus Uhlmann (1997). "Etude de modèles de fermeture au second ordre et contribution à la resolution numérique des écoulements turbulents compressibles", Thèse, Ecole centrale de Lion.

H. Stefan (2003). " Statistical Mechanics of turbulent Flows", Springer, Verlag Berlin. 